\title{
Pseudomonas putrefaciens as a Cause of Bacteremia in Humans
}

\author{
J. VANDEPITTE ${ }^{1 *}$ AND J. DEBOIS ${ }^{2}$ \\ Department of Microbiology, ${ }^{1}$ and Department of Dermatology, ${ }^{2}$ St.-Raphaël University Hospital, B-3000, \\ Leuven, Belgium
}

Received for publication 22 August 1977

\begin{abstract}
Pseudomonas putrefaciens has been presented as an opportunistic pathogen in a few isolations from humans. This is the first documented report of a local infection with $P$. putrefaciens complicated by invasion of the blood stream. The organism was twice isolated from the blood culture and once from the wound exudate of a 73-year-old woman hospitalized for extensive ulcerations on the legs. Parenteral ampicillin and gentamicin together with local antiseptic treatment led to an uneventful recovery.
\end{abstract}

Pseudomonas putrefaciens, a pseudomonaslike rod characterized by a strong production of $\mathrm{H}_{2} \mathrm{~S}$, is an unfamiliar organism for most clinical bacteriologists, although it has been reported with increasing frequency as a cause of infection in humans.

The first human isolates of a nonfermentative $\mathrm{H}_{2} \mathrm{~S}$-producing gram-negative rod with polar flagellation were described by King (11) in 1964, who gave them the provisional label of " $1 b$ ". In 1970 , it was recognized by Hugh (10) that these isolates were identical with $P$. putrefaciens. This bacterium, first isolated from butter (4) in 1931, was originally called Achromobacter putrefaciens. It was later transferred to the genus $P$ seudomonas (15). Similar bacteria, designated as $P$. rubescens (18) and Flavobacterium group 4 (17), are now believed to belong to the same species. More recently, its subdivision into two biotypes has been proposed $(13,19)$ and further documented $(9,24)$.

In 1972, Baumann et al. (1) proposed the new generic name Alteromonas for pseudomonaslike organisms with a deoxyribonucleic acid guanine + cytosine content between 43.2 and 48 mol\%, which is distinct from the higher value found in typical Pseudomonas species. This position was also held by Lee and associates (12), who examined a number of strains of $P$. putrefaciens and found their guanine + cytosine comprised between 44.7 and $54.7 \mathrm{~mol} \%$. They proposed, therefore, the inclusion of those strains in the new genus as Alteromonas putrefaciens Derby \& Hammer comb. nov.

In a previous paper, we have already drawn attention to the relatively frequent occurrence of $\boldsymbol{P}$. putrefaciens in pathological material from different sources (2), and a patient with a deep and long-standing infection of an ulcus cruris has been reported in greater detail (3). Most of our other isolates, however, and also many of those described by other authors $(2,5-9,11,16$, $17,19,20,22,23$ ), are of doubtful clinical significance, and some of them could easily be interpreted as accidental contaminants or transient colonizers of the skin or mucous membranes.

The present contribution seems important, since it describes a well-documented case in which the virulence of $P$. putrefaciens was demonstrated by its repeated isolation from the blood stream and from the infected tissue in a patient with ulcus cruris.

\section{CASE REPORT}

N.T., a 73-year-old woman, was admitted as an emergency case to our hospital on 24 August 1976. She suffered from fever and presented many confluent crusted ulcers on both lower legs.

In her previous history she mentioned a few similar episodes of fever, and lately she had experienced several attacks of recurrent thrombophlebitis. According to her physician, she had a moderate hypochromic anemia, for which she was treated with iron injections, and a mild renal insufficiency. On admission, she was febrile $\left(38.3^{\circ} \mathrm{C}\right)$ but conscious. On both legs, multiple superficial venous ulcers could be seen. The ulcerated area was covered with hemorrhagic crusts and was surrounded by a zone of exudative eczema and severe edema. On the back and the scapular regions of both arms there were extensive ecchymotic lesions. A brown discoloration of the skin involved the right gluteal region and the right thigh and knee. At no moment did the patient present any symptoms of gramnegative septicemia, such as hypotension or shock. 
On each of the 2 first days of hospitalization, venous blood was obtained for culture, and ivoth specimens yielded rapid growth of motile gramnegative rods that were identified as $P$. putrefaciens. Material was also obtained from the ulcerated surfaces on both legs. Only the exudate of the left leg was positive for $P$. putrefaciens, in mixed growth with $P$. aeruginosa. The material taken from the right side only grew the latter organism, together with Staphylococcus aureus. The ecchymotic lesions were interpreted as cutaneous manifestations of the septicemia, and this was confirmed by biopsy. Although the brown discoloration of the skin suggested iron infiltration secondary to therapy, no iron deposits could be demonstrated by skin biopsy.

After treatment with intravenous gentamicin and ampicillin, which were both active in vitro against the isolates of $P$. putrefaciens, the fever disappeared in a few days. The ulcers, however, only subsided after several weeks of local treatment with antiseptic dressings and creams. Subsequent blood cultures remained sterile, and repeated cultures of the ulcer exudate were all negative for $P$. putrefaciens. The patient was discharged on 4 November 1976 in good general condition and with completely healed ulcers.

\section{RESULTS}

From two blood cultures, taken on two successive days, and from the wound exudate of the ulcerations on the left leg, a strain was obtained that showed the growth characteristics and biochemical reactions of $P$. putrefaciens, as described in our previous paper (2). The most prominent feature of this organism is the heavy blackening of Kligler iron agar and triple sugar iron agar due to production of $\mathrm{H}_{2} \mathrm{~S}$.

A few unusual weakly or non- $\mathrm{H}_{2} \mathrm{~S}$-forming variants have been described $(14,22)$, but for the alerted bacteriologist they are sufficiently distinctive by their tan pigment, their deoxyribonuclease activity, and their unusual pattern of susceptibility to antimicrobial agents.

After one night of incubation, our isolates grew well on horse blood agar, forming large, smooth, and slightly mucoid circular colonies with a yellow-brown nondiffusible pigment. There was no hemolysis, but a diffuse greening of the medium was seen in the zone of heavy growth. On MacConkey agar, there was rapid and abundant growth in the form of tan, mucoid, convex, lactose-nonfermenting colonies. There was heavy growth on Kligler iron agar without acidification of the slant. The reaction of the butt, however, was masked by the heavy blackening of the medium. This pattern could have led to misidentification as anaerogenic Proteus,
Salmonella, or Citrobacter were it not for the strongly positive oxidase reaction.

The results of a series of other identification tests were identical for our three isolates and similar to those previously described (2).

On the basis of their weak saccharolytic activity, salt tolerance (assessed by growth in Trypticase soy broth containing $6.5 \% \mathrm{NaCl}$ ), and growth on salmonella-shigella agar, our isolates could be biotyped as belonging to Levin's group 2 (13). Most of the clinical isolates described by Holmes et al. (9) were also of group 2.

The results of the susceptibility tests (KirbyBauer method) revealed resistance to penicillin G, methicillin, lincomycin, and cephalothin, but sensitivity to sulfonamide, ampicillin, carbenicillin, streptomycin, kanamycin, gentamicin, tobramycin, amikacin, tetracycline, chloramphenicol, colistin, nalidixic acid, nitrofurantoin, co-trimoxazole, and erythromycin. This sensitivity pattern agrees well with those described by other authors $(7,9,20,23)$.

\section{DISCUSSION}

$P$. putrefaciens is a widely distributed saprophyte that has been isolated from stream-, lake-, sea-, and tap water, from soil, sewage, oil, salted butter, milk, fish, eggs, meat, oysters, and frogs $(4,5,9-13,15,18,20,24)$. It has been regarded as one of the most important organisms encountered in the spoilage of protein foods kept at chill temperatures (21). Contact of food with soil or water is probably responsible for contamination and spoilage.

$P$. putrefaciens seems to have a worldwide distribution in nature; most of the above-mentioned strains came from the United States and from the United Kingdom, but isolates have also been reported from wastewater in Tunisia $(J$. Kortleven, personal communication), from streamwater in Madagascar (5), and from seawater in Tahiti (5).

Human isolates of $P$. putrefaciens have been reported from a great variety of types of clinical material. The first human isolates were from the feces of Japanese patients with diarrhea (16). It has also been isolated from stools in the United States $(11,17)$ and from stools of patients in Madagascar (5) and Tunisia (J. Kortleven, personal communication). The wide spectrum of other clinical sources is apparent from the list of strains in the collection of the Communicable Disease Center $(11,23)$, sputum $(2,9,20$, $22,23)$, upper respiratory tract $(2,9)$, pleural fluid (9), spinal fluid $(9,11)$, ascitic fluid (22), pus and wounds $(2,8,9,17,19)$, varicose ulcers $(2,3,23)$, infected fracture $(8)$, bile (23), ear 
discharge $(8,9,11,23)$, and eye (11).

In most of these reports, the clinical circumstances in which the strains were isolated have not been documented, and some authors admitted that the organisms were without clinical significance or were even contaminants. According to von Graevenitz (23) and Holmes (8), $P$. putrefaciens appeared to play a pathogenic role mainly in cases of otitis media. Among our own previous isolates (2), only the strain that was repeatedly recovered from the depth of a varicose leg ulcer appeared to play an etiological role (3). It might be more than coincidence that in the present patient the same organism was again isolated from the same site. Both patients lived in poor hygienic conditions, and their ulcers may have been contaminated by repeated contact with dirt and soil. The psychrophylic nature of $P$. putrefaciens makes it particularly well adapted to thrive in the devitalized necrotic tissue of venous ulcers.

The main interest of our report is the evidence that, in rare instances, this opportunistic, lowgrade pathogen can invade the bloodstream and cause systemic infection, even in a patient without gross underlying disease or immunological defects.

Diagnostic bacteriologists should be aware of the existence of this organism, which is of rather common occurrence and easy to recognize on the basis of a few simple characteristics.

\section{ACKNOWLEDGMENTS}

We thank B. Holmes of the Computer Trials Laboratory, Central Public Health Laboratory, Colindale, London, who kindly confirmed the identity and the biotype of one of our isolates.

\section{LITERATURE CITED}

1. Baumann, L., P. Baumann, M. Mandel, and R. D. Allen. 1972. Taxonomy of aerobic marine eubacteria. J. Bacteriol. 110:402-429.

2. Debois, J., H. Degreef, J. Vandepitte, and J. Spaepen. 1975. Pseudomonas putrefaciens as a cause of infection in humans. J. Clin. Pathol. 28:993-996.

3. Degreef, H., J. Debois, and J. Vandepitte. 1975. Pseudomonas putrefaciens as a cause of infection of venous ulcers. Dermatologica 151:296-301.

4. Derby, H. A., and B. W. Hammer. 1931. Bacteriology of butter. IV. Bacteriological studies in surface taint butter. Iowa Agric. Home Econ. Exp. Stn. Res. Bull. 145:387-416.

5. Fourquet, R., P. Coulanges, J. Goasguen, and J. L. Boehrer. 1975. Premières souches de Pseudomonas putrefaciens isolées à Madagascar. Arch. Inst. Pasteur Madagascar 44:49-55.
6. Gilardi, G. L. 1971. Characterization of Pseudomonas species isolated from clinical specimens. Appl. Microbiol. 21:414-419.

7. Gilardi, G. L. 1971. Antimicrobial susceptibility as a diagnostic aid in the identification of nonfermenting gram-negative bacteria. Appl. Microbiol. 22:821-823.

8. Gilardi, G. L. 1972. Infrequently encountered Pseudomonas species causing infection in humans. Ann. Intern. Med. 77:211-215.

9. Holmes, B., S. P. Lapage, and H. Malnick. 1975. Strains of Pseudomonas putrefaciens from clinical material. J. Clin. Pathol. 28:149-155.

10. Hugh, R. 1970. A practical approach to the identification of certain non-fermentative gram-negative rods encountered in clinical specimens. Public Health Lab. 28:168-187.

11. King, E. O. 1964. The identification of unusual pathogenic gram-negative bacteria. National Communicable Disease Center, Atlanta.

12. Lee, J. V., D. M. Gibson, and J. M. Shewan. 1977. A numerical taxonomic study of some Pseudomonas-like marine bacteria. J. Gen. Microbiol. 98:439-451.

13. Levin, R. E. 1972. Correlation of DNA base composition and metabolism of Pseudomonas putrefaciens isolates from food, human clinical specimens and other sources. Antonie van Leeuwenhoek J. Microbiol. Serol. 38:121-127.

14. Levin, R. E. 1975. Characteristics of weak- $\mathrm{H}_{2} \mathrm{~S}$-producing isolates of Pseudomonas putrefaciens from human infections. Antonie van Leeuwenhoek J. Microbiol. Serol. 41:569-574.

15. Long, H. F., and B. W. Hammer. 1941. Distribution of Pseudomonas putrefaciens. J. Bacteriol. 41:100-101.

16. Minagawa, M. 1963. Studies on the strains closely related to Vibrio parahaemolyticus and reddish brown pigment producing Pseudomonas isolated from the stools of patients of acute enteritis. Annu. Rep. Inst. Food Microbiol. 16:9-23.

17. Pedersen, M. M., E. Marso, and M. J. Pickett. 1970. Non-fermentative bacilli associated with man. III. Pathogenicity and antibiotic susceptibility. Am. J. Clin. Pathol. 54:178-192.

18. Pivick, H. 1955. Pseudomonas rubescens, a new species from soluble oil emulsions. J. Bacteriol. 70:1-6.

19. Riley, P. S., H. W. Tatum, and R. E. Weaver. 1972. Pseudomonas putrefaciens isolated from clinical specimens. Appl. Microbiol. 24:798-800.

20. Rosenthal, S. L., J. H. Zuger, and E. Apollo. 1975. Respiratory colonization with Pseudomonas putrefaciens after near-drowning in salt water. Am. J. Clin. Pathol. 64:382-384.

21. Shewan, J. M. 1974. The biodeterioration of certain proteinaceous food-stuffs at chill temperatures. In B. Spender (ed.), Industrial aspects of biochemistry, North-Holland Publishing Co., London.

22. von Graevenitz, A. 1976. Detection of unusual strains of gram-negative rods through the routine use of a deoxyribonuclease-indole medium. Mt. Sinai J. Med. 43:727-735.

23. von Graevenitz, A., and G. Simon. 1970. Potentially pathogenic, nonfermentative, $\mathrm{H}_{2} \mathrm{~S}$-producing gram-negative rod (1b). Appl. Microbiol. 19:176.

24. Williams, J. L., and R. E. Levin. 1975. Bacteriocin typing of Pseudomonas putrefaciens from food, human clinical specimens and sources. Antonie van Leeuwenhoek J. Microbiol. Serol. 41:97-100. 